\title{
VLF-LF radio signals collected at Bari (South Italy): a preliminary analysis on signal anomalies associated with earthquakes
}

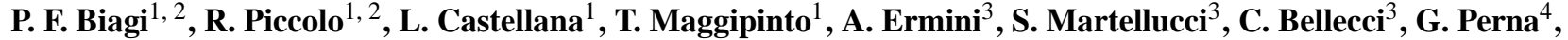 \\ V. Capozzi ${ }^{4,2}$, O. A. Molchanov ${ }^{5}$, M. Hayakawa ${ }^{6}$, and K. Ohta ${ }^{7}$ \\ ${ }^{1}$ Department of Physics, University of Bari, Via Amendola, 173, 70126 Bari, Italy \\ ${ }^{2}$ INFM-Unity of Bari, Via Amendola, 173, 70126 Bari, Italy \\ ${ }^{3}$ Department of Engineering of Enterprise, University of Roma Tor Vergata, Via di Tor Vergata, 00133 Rome, Italy \\ ${ }^{4}$ Department of Biomedical Sciences, University of Foggia, Via L. Pinto, 71100 Foggia, Italy \\ ${ }^{5}$ United Institute of the Earth's Physics, Russian Academy of Science, Bolshaya Gruzinskaya 10, 123995 Moscow, Russia \\ ${ }^{6}$ Dept. of Electronic Engineering, Univ. of Electro-Communications, 1-5-1 Chofugaoka, Chofu City, Tokyo 182-8585, Japan \\ ${ }^{7}$ Department of Electronics Engineering, Chubu University, 1200 Matsumoto-cho, Kasugai Aichi, 487-8501, Japan
}

Received: 22 June 2004 - Revised: 28 October 2004 - Accepted: 29 October 2004 - Published: 3 November 2004

Part of Special Issue "Precursory phenomena, seismic hazard evaluation and seismo-tectonic electromagnetic effects"

\begin{abstract}
At the beginning of 2002 an OmniPAL receiver was put into operation at the Department of Physics of Bari University (Southern Italy). The electric field strength of five VLF-LF signals transmitted from United Kingdom $(\mathrm{f}=16 \mathrm{kHz})$, France $(\mathrm{f}=20.9 \mathrm{kHz})$, Germany $(\mathrm{f}=23.4 \mathrm{kHz})$, Iceland $(\mathrm{f}=37.5 \mathrm{kHz})$ and Italy $(\mathrm{f}=54 \mathrm{kHz})$ has been monitoring with a $5 \mathrm{~s}$ sampling frequency. In a first step we reduced the amount of the data taking one datum each 10 min (mean of the \pm 5 min raw data) and then we smoothed these data by a running adjacent averaging over 7 days. Analysing the trends we obtained, we revealed at first in the signal from the Italian transmitter two clear intensity decreases in April 2002 and in August-September 2002. At these times we observed earthquakes with $\mathrm{M}=4.3$ and $\mathrm{M}=5.6$ respectively near the transmitter-receiver path and a precursory effect in the previous decreases appeared. Then, we noted that all of the five radio trends in the time interval March 2002-February 2003 are more disturbed than in other periods; in particular an evident simultaneous decrease appears in JanuaryFebruary 2003. We propose that these disturbances are related to general excitation of the margin between the African and European plates. In a second step we examined the terminator time (evening) changes for the Italian transmitter in July-September 2002, and we found significant deviations from the mean value at the end of August, which is supportive for some precursory ionospheric signature of earthquakes.
\end{abstract}

Correspondence to: P. F. Biagi

(biagi@fisica.uniba.it)

\section{Introduction}

For many years there has been carried out research on the interaction between seismic activity and disturbances in radiobroadcasts. One of the first results was obtained using $18 \mathrm{MHz}$ receivers on the occasion of the great $(\mathrm{M}=8.5)$ Chilean earthquake of 22 May 1960 (Warwick et al., 1982). The receivers were a part of the network used for studying cosmic noise. Later, pre-seismic disturbances in VLF radio signals lying in the $3-30 \mathrm{kHz}$ frequency band, have been presented mainly by Japanese, Russian and Greek researchers (Hayakawa and Sato, 1994; Hayakawa et al., 1996, 2002; Morgounov et al., 1994; Molchanov and Hayakawa, 1998; Nomikos et al., 1997; Vallianatos and Nomikos, 1998). Recently, pre-seismic disturbances on LF $(150-250 \mathrm{kHz})$ radio broadcasts were proposed mainly by Italian researchers (Bella et al., 1998; Biagi, 1999; Biagi et al., 2001a, b; Biagi and Hayakawa, 2002). From 2000 a scientific cooperation among the Japanese, Russian and Italian teams started. In this framework, an OmniPAL receiver able to measure the electric field strength and the phase of five VLF-LF radiosignals was put into operation at Bari (South Italy) at the beginning of 2002. Here we present some preliminary analysis results of the data collected till now.

\section{Observation of radio signals and analysis methods}

The OmniPAL receiver is located at the Department of Physics of Bari University and is connected to a computer by a digital card. The electric antenna and the GPS 


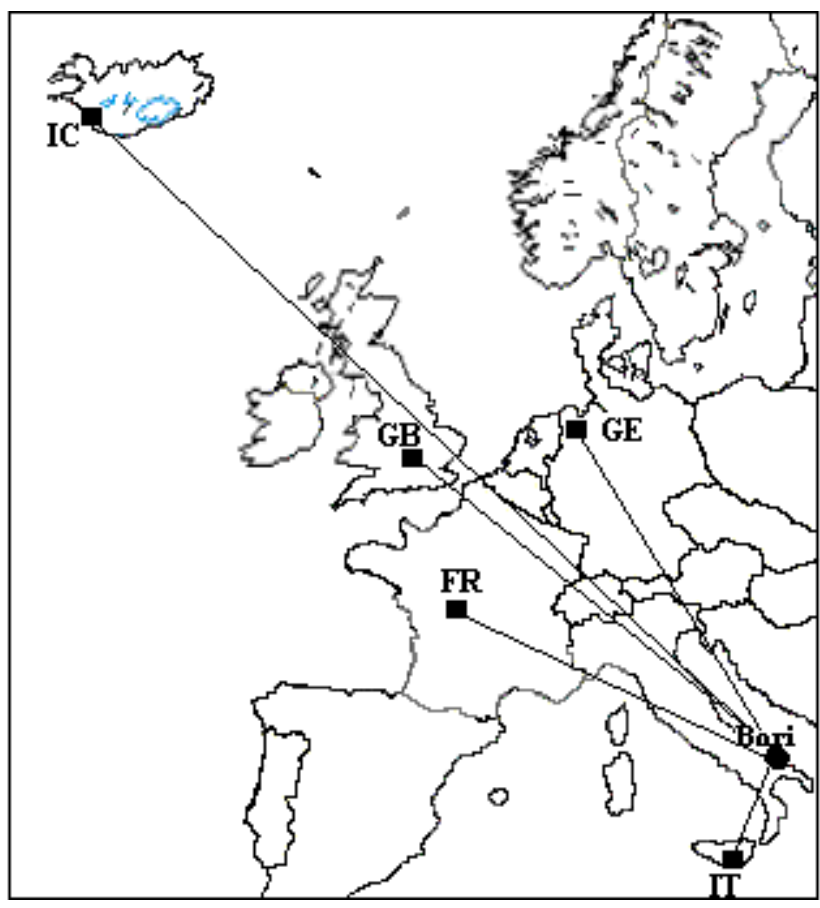

Fig. 1. Map showing the location of the receiver (Bari) and of the VLF/LF transmitters: GB ( $\mathrm{f}=16 \mathrm{kHz}$, United Kingdom), FR ( $\mathrm{f}=20.9$ $\mathrm{kHz}$, France), GE ( $\mathrm{f}=23.4 \mathrm{kHz}$, Germany), IC ( $\mathrm{f}=37.5 \mathrm{kHz}$, Iceland) and IT ( $\mathrm{f}=54 \mathrm{kHz}$, Sicily, Italy). The different radio-paths are plotted.

sensor are installed at the roof of the Department. On the basis of the best reception at the receiver and taking into account the more convenient (in relation to the seismic activity) radio paths we selected the transmitters: GB ( $\mathrm{f}=16 \mathrm{kHz}$, United Kingdom), FR ( $\mathrm{f}=20.9 \mathrm{kHz}$, France), GE $(\mathrm{f}=23.4 \mathrm{kHz}$, Germany), IC ( $\mathrm{f}=37.5 \mathrm{kHz}$, Iceland) and IT $(\mathrm{f}=54 \mathrm{kHz}$, Sicily, Italy). The transmitters-receiver distance ranges from $500 \mathrm{~km}$ to $3500 \mathrm{~km}$. Figure 1 shows the location of the VLF/LF transmitters and of our receiver. A $5 \mathrm{~s}$ sampling rate was selected. Unfortunately the phase data are not available due to malfunction of the phase card of the equipment; so we analysed only the electric field strength data. As an example, two raw time series collected during one week are shown in Fig. 2. The features of the signals are the standard ones with high level at night time and low level at day time.

In a first analysis we studied the long-term variations of the electric field strength of the radio signals. For this purpose, we reduced at first the amount of the data taking one datum each $10 \mathrm{~min}$ that is the mean of the raw data $5 \mathrm{~min}$ before and $5 \mathrm{~min}$ after the datum. It must be noted that this procedure does not change the features of the signals. Then we smoothed the data by a running adjacent averaging over 7 days. Figure 3 shows the one datum each 10 min time series and the relative smoothed time series of the IC radio-signal from the beginning (February 2002) to January 2004. Unfortunately, some interruption exists mainly that occurred in the

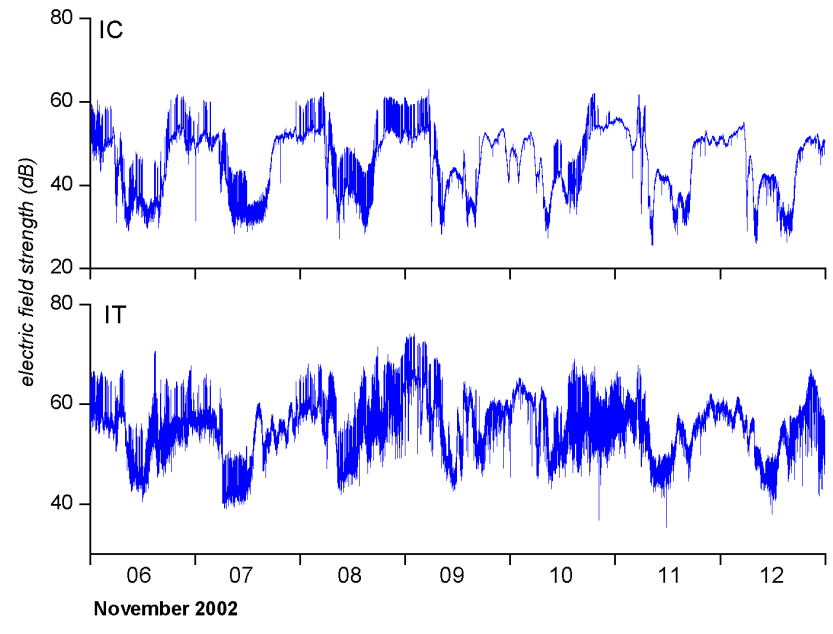

Fig. 2. Raw data on time series of the electric field strength of IC and IT radio-signals collected in the time interval of 6-12 November, 2002.

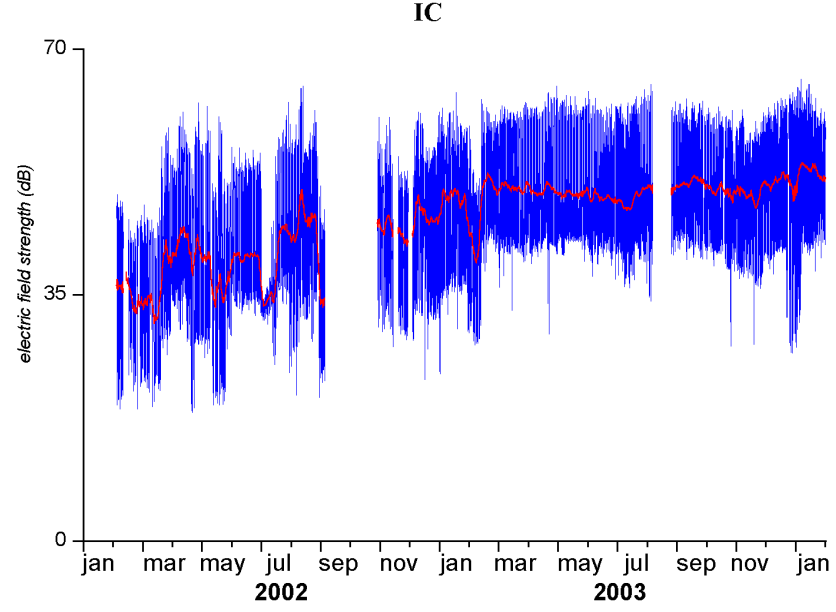

Fig. 3. Electric field strength of the IC radio-signal collected from February 2002 to January 2004. Every datum of the blue trend is the mean of the raw data $5 \mathrm{~min}$ before and $5 \mathrm{~min}$ after the datum. The red line represents the 7 days running adjacent averaging of the blue trend.

period September-October 2002 related to human activity at the place where the receiver antenna is located. Of course, such an interruption exists in the trends of all the five radiosignals.

In addition to the above field intensity estimation, we adopted in the period July-September 2002 the terminator time method proposed by Hayakawa et al. (1996).

\section{Observational results}

As it is concerned with the first analysis, Fig. 4 shows the trends of the five smoothed radio-signals we obtained. Some disturbances clearly related to the transmitters were corrected. From Fig. 4 it is possible to note: (a) an anomalous 


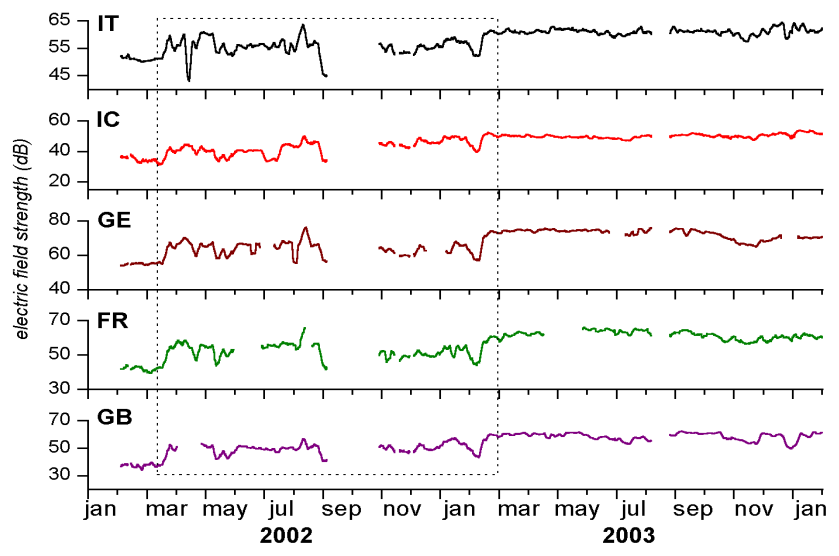

Fig. 4. Time series of the electric field strength of the five radio signals obtained with a 7 days running adjacent averaging. The parts of the trends covered by the dotted lines, are more disturbed with respect to the remaining parts.

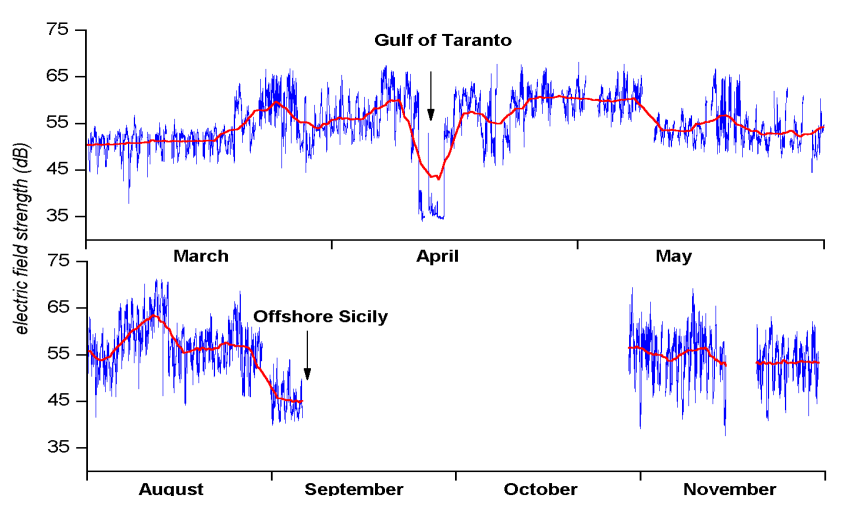

Fig. 5. (a) Enlargement of the trend of the IT radio-signal in the period March-May 2002. The arrow indicates the occurrence of the gulf of Taranto main earthquakes. (b) Enlargement of the same trend in the period July-November 2002. The arrow indicates the occurrence of the offshore Sicily main shock. Every datum of the blue trends is the mean of the raw data $5 \mathrm{~min}$ before and $5 \mathrm{~min}$ after the datum. The red lines represent the 7 days running adjacent averaging of the relative blue trends.

decrease in the IT radio-signal alone during April 2002; (b) an anomalous decrease particularly evident in the IT radiosignal, starting at the end of August 2002; (c) that the part (marked by dotted lines in Fig. 4) from March 2002 to February 2003, is more disturbed in all the trends as compared to the remaining parts; moreover, a transient (January-February 2003) simultaneous decrease in all the trends appears. An enlargement of the decreases pointed out in items (a) and (b) is shown in Figs. 5a and 5b, respectively. On 17 April 2002 three earthquakes with $\mathrm{M}=4.3-3.0$ happened in short sequence in the gulf of Taranto and an aftershock $(M=3.4)$ occurred on 27 April; on 6 September 2002 an earthquake with $\mathrm{M}=5.6$ occurred offshore Sicily starting an intense seismic sequence till the end of October. The occurrence of the main shocks is indicated in Fig. 5; the location of the earthquakes is shown in the map of Fig. 6. In order to give any

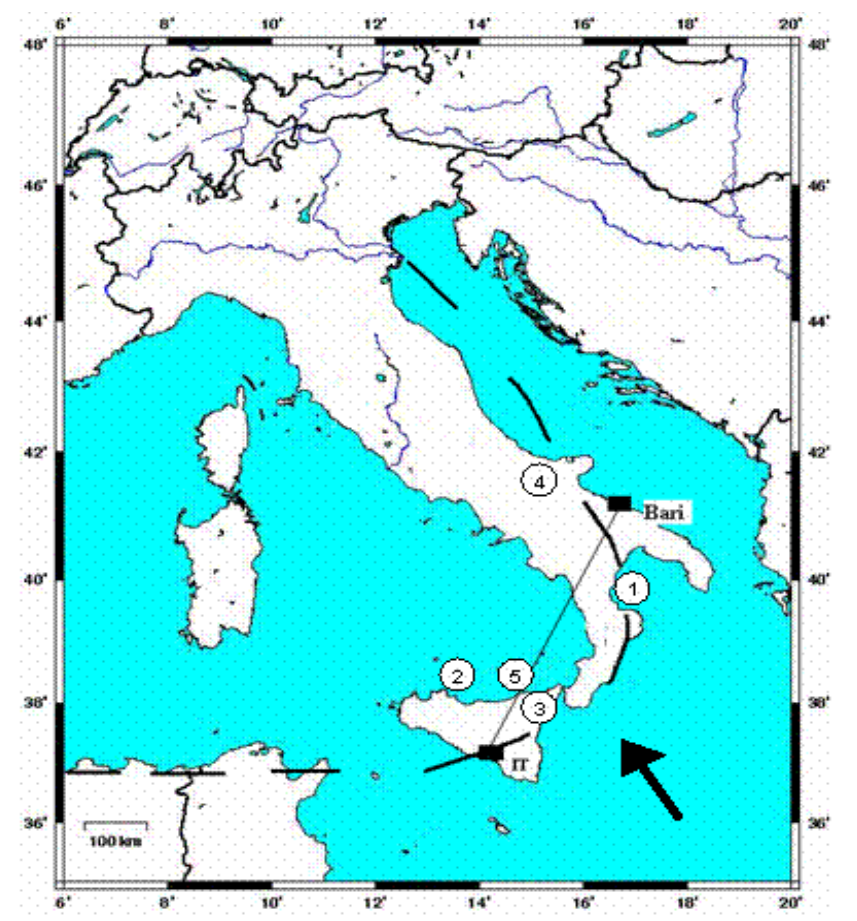

Fig. 6. Map showing the location of: (1) the gulf of Taranto earthquakes, (2) the offshore Sicily seismic crisis, (3) the Etna volcano, (4) the Molise seismic crisis, (5) the gas exhalation at Eolian islands. The black line indicates the IT-Bari radio-path. The black dashed line represents approximately the margin between the African plate and the European plate. An arrow indicates the direction of the relative movement of the two plates.

further support to the anomaly at the end of August 2002 in the IT radio-signal, we examined the evening terminator times as proposed by Hayakawa at al. (1996). The result of this study is shown in Fig. 7. Also the geomagnetic activity represented by the planetary magnetic $k_{p}$ indices was examined. We identified increases of the indices in the time interval 17-20 April 2002 and in the time interval 4-7 September 2002. But similar increases appeared also in other time interval during 2002 and 2003.

\section{Discussion}

Concerning with the first analysis we made, let us first consider the anomalous decrease in Fig. 5a and the possible correlation with the gulf of Taranto earthquakes. We note that: (a) the decrease starts $4-5$ days before the increase of the geomagnetic activity; (b) the decrease appears only in the IT trend (Fig. 4); (c) the earthquakes occurred at a distance of $70 \mathrm{~km}$ from the IT radio-path (Fig. 6); (d) the seismicity of the zone is characterized by earthquakes with very small magnitude (1.5-2.5) and so the previous events are rare ones and they were the strongest in the last years. In conclusion the connection between these earthquakes and the anomalous decrease in IT radio-signal seems reasonable. In this point of 


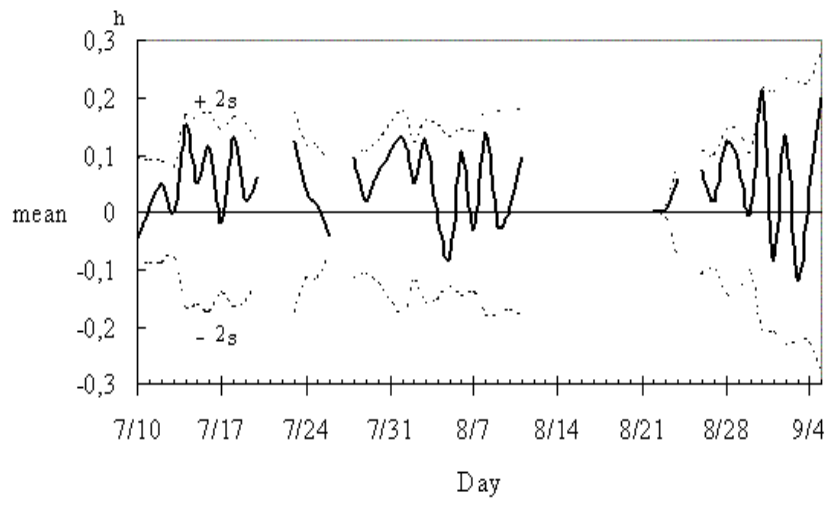

Fig. 7. Temporal evolution of terminator time (evening) change during the period July-September, 2002 for the IT-Bari path. The zero level indicates the mean value and the full line the current deviation from the mean. The $\pm 2 \sigma$ ( $\sigma$ is the standard deviation) lives are indicated as broken line.

view, the duration of the radio anomaly is about 7 days and a premonitory phase of 3 days exists.

Now let us consider the anomalous decrease in Fig. 5b and its possible connection with the offshore Sicily seismic sequence. At first it must be noted that the data collection was interrupted (Sect. 2) on 5 September that is one day before the beginning of the seismic sequence; so, it is not possible to have the complete features of the anomalous decrease. But, we can note that: (a) the decrease starts on 29 August that is 6 days before the beginning of the geomagnetic activity increase; (b) the quoted decrease is particularly clear only in the IT trend (Fig. 4); (c) the seismic events are located in a zone at a distance less than $100 \mathrm{~km}$ from the IT radiopath (Fig. 6); (d) the sequence was very intense and the main shock was the largest ever occurred in the seismo-active area offshore Sicily in the last years. Taking into account these remarks, our opinion is that the connection between the offshore Sicily earthquakes and the anomalous decrease in IT radio-signal can be proposed. In this point of view, a premonitory phase of 8 days exists in the radio anomaly.

In both the previous cases, some perturbation seeming to occur in a limited region of the atmosphere-ionosphere produced by the earthquake preparation could justify the radio anomalies observed.

Now, let us consider the disturbed part (Fig. 4) in all the trends, including also the simultaneous decrease during January-February 2003, pointed out in the previous section. We did not find a substantial difference of the geomagnetic activity $\left(k_{p}\right.$ indices) during the time interval of the quoted disturbances respect to the geomagnetic activity during the other time intervals reported in the Fig. 4. On the contrary an unusual very intense tectonic activity took place in South Italy during the time interval of the quoted disturbances. After the quoted gulf of Taranto earthquakes (April 2002) and the offshore Sicily seismic sequence (September-October 2002), on 27 October the Etna volcano started a strong eruption, on 31 October an earthquake with $\mathrm{M}=5.5$ happened in the Molise region starting an intense seismic crisis and at the beginning of November anomalous gas exhalations were observed at the Eolian islands. The location of these activities is shown in Fig. 6. Unfortunately, these last three activities happened in the period of interruption in data collecting (Sect. 2). Biagi et al. (2004) presented possible disturbances in a LF $(189 \mathrm{kHz})$ radio broadcast transmitted from Sicily and related to such activities. In Fig. 6 the margin between the African and the European plates is also drawn. The African plate is moving northwestward against the Eurasian plate with a velocity of about $6 \mathrm{~mm}$ per year and this relative plate motions create a compressional tectonic environment. It seems evident that all the previous activities are related to some changes occurred in this relative movement. So, all the plates margin was excited for a long time and, at this time, the atmosphere-ionosphere could be perturbed in a wide zone. So, the disturbed part existing in all the trends could be related to these perturbations. In particular, the simultaneous decrease of the radio trends during JanuaryFebruary 2003 could be related to a particular clear perturbation in the atmosphere-ionosphere produced by a large excitation of the plates margin, probably the final step of the change process. It must be noted that on 21 May 2003 a large $(M=6.8)$ earthquake happened in Algeria, at the west border of the quoted margin, probably as the final adjustment of all the tectonic process occurred in 2002-2003.

Finally, Fig. 7 illustrates the temporal evolution of the terminator time changes in the period July-September 2002 for the IT-Bari propagation path. The mean and standard deviation $(\sigma)$ are estimated by using the data during 7 days before the current day. The zero line indicates the mean value of the evening terminator time and $\pm 2 \sigma$ lives are also given in dotted lines in the Fig. 7. The full live is the result on the current day. It is found that there is an anomalous change in the terminator time of exceeding $2 \sigma$ live on 31 August 2002, which suggests that there is present any definite precursory ionospheric perturbations of the earthquakes.

\section{Conclusions}

The results we presented seem to confirm a good sensitivity of VLF-LF radio waves propagation to the seismicity. Moreover, the results seems to indicate a wider sensitivity that is to large tectonic processes as plates margin movements.

Acknowledgements. Two of the authors (M. Hayakawa and K. Ohta) are grateful to the Japan Society of Promotion of Science (JSPS) for its Grants-in-Aids (\#15403012) and the Mitsubishi Foundation for its support.

The seismic data used in this paper were obtained by the INGV (Istituto Nazionale di Geofisica e Vulcanologia) web site (http://www.ingv.it).

Edited by: M. Contadakis

Reviewed by: F. Vallianatos and another referee 


\section{References}

Bella, F., Biagi, P. F., Caputo, M., Cozzi, E., Della Monica, G., Ermini, A., Plastino, W., and Sgrigna, V.: Field strength variations of LF radio waves prior to earthquakes in Central Italy, Phys. Earth Planet. Inter., 105, 279-286, 1998.

Biagi, P. F.: Seismic effects on LF radiowaves, In: Atmospheric and Ionospheric Electromagnetic Phenomena Associated with Earthquakes, edited by Hayakawa, M., TERRAPUB, Tokyo, 535-542, 1999.

Biagi, P. F., Piccolo, R., Ermini, A., Martellucci, S., Bellecci, C., Hayakawa, M., Capozzi, V., and Kingsley, S. P.: Possible earthquake precursors revealed by LF radio signals, Nat. Haz. Earth Sys. Sci., 1, 1-2, 99-104, 2001a.

Biagi, P. F., Piccolo, R., Ermini, A., Martellucci, S., Bellecci, C., Hayakawa, M., and Kingsley, S. P.: Disturbances in LF radiosignals as seismic precursors, Annali di Geofisica, 44, 5/6, 10111019, $2001 b$.

Biagi, P. F. and Hayakawa, M.: Possible premonitory behaviour of LF radiowaves on the occasion of the Slovenia earthquakes ( $M=5.2-6.0-5.1$ ) occurred on March-May 1998, in Seismo Electromagnetics: Lithosphere-Atmosphere-Ionosphere Coupling, edited by Hayakawa, M. and Molchanov, O., TERRAPUB, Tokyo, 249-253, 2002.

Biagi, P. F., Piccolo, R., Castellana, L., Ermini, A., Martellucci, S., Bellecci, C., Capozzi, V., Perna, G., Molchanov, O., and Hayakawa, M.: Variations in a LF radio signal on the occasion of the recent seismic and volcanic activity in Southern Italy, Phys. Chem. Earth, 29, 4/9, 551-557, 2004.

Hayakawa, M. and Sato, H.: Ionospheric perturbations associated with earthquakes, as detected by subionospheric VLF propagation, in: Electromagnetic Phenomena Related to Earthquake Prediction, edited by Hayakawa, M. and Fujinawa, Y., TERRAPUB, Tokyo, 391-397, 1994.
Hayakawa, M., Molchanov, O. A., Ondoh, T. and Kawai, E.: Anomalies in the subionospheric VLF signals for the 1995 Hyogo-ken earthquake, J. Phys. Earth, 44, 413-418, 1996.

Hayakawa, M., Molchanov, O. A., Shima, N., Shvets, A. V., and Yamamoto, N.: Wavelet analysis of disturbances in subionospheric VLF propagation correlated with earthquakes, in Seismo Electromagnetics: Lithosphere-Atmosphere-Ionosphere Coupling, edited by Hayakawa, M. and Molchanov, O., TERRAPUB, Tokyo, 223-228, 2002.

Molchanov, O. A. and Hayakawa, M.: Subionospheric VLF signal perturbations possibly related to earthquakes, J. Geophys. Res., 103, 17 489-17 504, 1998.

Morgounov, V. A., Ondoh, T., and Nagai, S.: Anomalous variation of VLF signals associated with strong earthquakes $(M \geq 7.0)$, in: Electromagnetic Phenomena Related to Earthquake Prediction, edited by: Hayakawa, M. and Fujinawa, Y., TERRAPUB, Tokyo, 409-428, 1994.

Nomikos, K., Vallianatos, F., Kaliakatsos, J., Sideris, S., and Bakatsakis, M.: Latest aspects of telluric and electromagnetic variations associated with shallow and intermediate depth earthquakes in South Aegean, Annali di Geofisica, XI, 2, 361-374, 1997.

Vallianatos, F. and Nomikos, K., Seismogenic radioemissions as precursors to earthquakes in Greece, Phys. Chem. Earth, 23, 910, 953-959, 1998.

Warwick, J. W., Stoker, C., and Meyer, T. R.: Radio emission associated with rock fracture: possible application for the great Chilean earthquake of 22 May 1960, J. Geophys. Res., 87, 28512859, 1982. 